\title{
Competência social e empatia: um estudo sobre resiliência com crianças em situação de pobreza
}

\author{
Alessandra Marques Cecconello \\ Sílvia Helena Koller ${ }^{1}$ \\ Universidade Federal do Rio Grande do Sul
}

Resumo

O objetivo desse estudo foi avaliar a competência social e a empatia em crianças escolares que vivem em situação de pobreza. Participaram da pesquisa 100 crianças, de ambos os sexos, com idades entre seis e nove anos. Os instrumentos utilizados foram o Teste das Histórias Incompletas (THI) (Mondell \& Tyler, 1981) e a Escala de Empatia (Bryant, 1982), que avaliaram, respectivamente, competência social e empatia. Os resultados demonstraram que as meninas são mais competentes socialmente e mais empáticas do que os meninos. Da mesma forma, as crianças mais empáticas tendem a ser mais competentes socialmente do que as outras. De um modo geral, os dados obtidos enfatizam a importância dessas duas características como fatores de proteção, contribuindo para a resiliência e adaptação.

Palavras-chave: Competência social, empatia, resiliência, pobreza. 


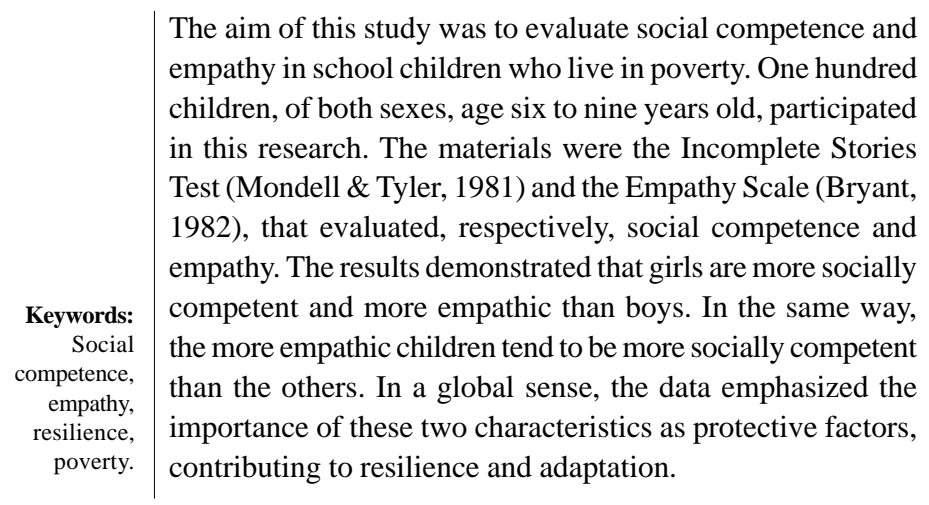

$\mathrm{O}$ estudo do desenvolvimento humano, com ênfase nos processos evolutivos saudáveis que caracterizam a resiliência, vem sendo o foco central no campo das pesquisas em Psicologia do Desenvolvimento. Dentro de uma perspectiva ecológica (Bronfenbrenner, 1979/1996; Bronfenbrenner \& Morris, 1998), o desenvolvimento é considerado como um envolvimento dinâmico de interações entre o homem e o ambiente. Nesta perspectiva, atualmente, várias pesquisas apontam para aspectos do meio-ambiente que devem ser considerados para o entendimento dos processos adaptativos dos indivíduos no curso de suas vidas (Masten \& Coatsworth, 1995; Rutter, 1996). Entre eles, encontram-se os fatores de proteção e de risco, que estão relacionados com eventos de vida, recursos, disposições ou demandas e que podem proteger o indivíduo de eventos estressantes e/ou levá-los a desencadear patologias (Bronfenbrenner \& Morris, 1998; Garmezy \& Masten, 1994). 
Apesar dessa ênfase, observa-se, entretanto, uma grande lacuna na Psicologia com relação a estudos sobre o desenvolvimento de crianças e adolescentes em situações adversas (Emde, 1994; Fischer, 1993; Huston, McLoyd, \& Coll, 1994; Hutz \& Koller, 1997). A pobreza, por exemplo, é considerada como um tipo de ameaça constante, que aumenta a vulnerabilidade da criança, pois pode causar subnutrição, privação social e desvantagem educacional (Zimmerman \& Arunkumar, 1994). Pesquisadores ressaltam que crescer na pobreza consiste numa ameaça ao bem-estar da criança e numa limitação de suas oportunidades de desenvolvimento. Mais do que essa limitação, a miséria econômica oriunda de fatores políticos e sociais que operam num nível macrossistêmico ecológico pode afetar o desenvolvimento da criança quando conduz à miséria afetiva. A miséria econômica é um fator de risco que em determinadas situações não vem desacompanhado: ela permeia a relação conjugal, contribuindo para o aumento da incidência de conflitos entre os pais e produzindo um efeito direto no relacionamento desses pais com a criança. Desta forma, ocasiona o que alguns autores denominam como miséria afetiva (Hutz, Koller \& Bandeira, 1996; Nunes, 1994; Pierre \& Layzer, 1998).

Nesse sentido, muitas crianças crescem e se desenvolvem em contextos e situações que constituem ameaças à sua saúde psicológica. Ao enfrentar situações de stress e risco no seu cotidiano, podem apresentar distúrbios emocionais e problemas de conduta, sendo vulneráveis. Entretanto, observa-se que nem todas as crianças que vivenciam as mesmas situações de risco apresentam problemas. Pelo contrário, algumas delas conseguem adaptar-se e superar essas situações, demonstrando, entre outras habilidades, competência social (Cecconello, 1999). Estas crianças são chamadas resilientes.

Resiliência é um conceito relacionado à adaptação e consiste em variações individuais em resposta aos fatores de risco (Rutter, 1996). Para Garmezy (1996), resiliência pode ser definida a partir da compreensão das consequiências da exposição de adultos e crianças a fatores de risco: alguns podem desenvolver problemas, outros podem superar as adversidades, adaptando-se ao contexto. Vulnerabilidade refere-se a uma predisposição individual que potencializa os efeitos 
de um estressor (Garmezy \& Masten, 1994). Ao contrário da resiliência, vulnerabilidade caracteriza-se por respostas mal-adaptadas que resultam em conseqüências negativas para o desenvolvimento psicológico (Zimmerman \& Arunkumar, 1994).

Interagindo com os fatores de risco encontram-se os fatores de proteção, que se distinguem como características potenciais na promoção de resiliência. Garmezy e Masten (1994) identificaram três fatores de proteção em indivíduos: (1) características individuais, como auto-estima, inteligência, capacidade para resolver problemas e competência social; (2) apoio afetivo transmitido pelas pessoas da família, através de um vínculo positivo com os cuidadores; (3) apoio social externo, provido por outras pessoas significativas, como escola, igreja e grupos de ajuda.

O conjunto destes fatores, em situações de stress, serve como um recurso que auxilia o indivíduo a interagir com os eventos de vida e conseguir bons resultados, evitando consequiências negativas. Neste sentido, a competência social é vista como um fator de proteção para o indivíduo, pois está relacionada com a capacidade para uma adaptação favorável (Cecconello, 1999). Competência social é uma característica individual [um dos três fatores mencionados por Garmezy e Masten (1994)] que leva em conta a interação do indivíduo com os integrantes dos outros dois fatores: a família e outras pessoas significativas. Vários autores definem competência social dentro deste pressuposto (Coble, Gantt, \& Mallinckrodt, 1996; Del Prette \& Del Prette, 1999; Kliewer, 1991; Tyler, 1984; Zigler \& Trickett, 1978).

Zigler e Trickett (1978) destacam dois aspectos para competência social. Primeiro, o sucesso da pessoa ao perceber as expectativas do ambiente, ou seja, que tipo de comportamento se faz necessário em determinada situação. Segundo, a pessoa deve demonstrar características importantes para seu desenvolvimento, como por exemplo auto-estima e auto-eficácia. Baseados nesse pressuposto, estudos sobre resiliência têm operacionalizado medidas de competência social através da observação de comportamentos manifestos, pois supõem que por trás desses comportamentos existem boas habilidades e estratégias eficazes de enfrentamento (Kliewer, 1991; Luthar, 1991). 
Kliewer (1991) utiliza o termo competência social como um entendimento de quais comportamentos são apropriados para determinadas circunstâncias e um esforço no sentido de adequar o comportamento à situação. Para o autor, crianças socialmente competentes são hábeis para entender as normas sociais, para a interação com pares e adultos, e hábeis para regular suas emoções, especialmente, as emoções negativas.

Nesse sentido, também, Del Prette e Del Prette (1999) definem a competência social como a capacidade da pessoa para apresentar um comportamento que possa atingir os objetivos de uma situação interpessoal, mantendo uma relação com o interlocutor através de equilíbrio de poder e de trocas positivas. Além disso, também destacam a importância de desenvolver características positivas para o crescimento pessoal, como auto-estima, e o respeito pelos direitos humanos socialmente estabelecidos.

Da mesma forma, Tyler (1984) define competência social como a maneira como o indivíduo interage com os eventos de vida, tanto num sentido de resolução de problemas, como num sentido de auto-realização. $\mathrm{O}$ autor destaca três características importantes para a competência social: (1) confiança otimista nas pessoas e no mundo; (2) autoeficácia, auto-avaliação positiva, e controle dos eventos de vida pessoal; (3) iniciativa, através do estabelecimento de objetivos realistas e esforços no sentido de alcançá-los. Além disso, uma capacidade para desfrutar do êxito, sofrer com os fracassos e construir com base em ambos.

Dentro desta perspectiva, um indivíduo competente é capaz de acreditar em suas potencialidades e demonstrar sentimentos positivos com relação a si mesmo. Além disso, é capaz de estabelecer metas e traçar estratégias para conseguir bons resultados, mesmo quando fracassa (Cecconello, 1999).

Parece haver concordância entre os pesquisadores do tema a respeito da importância da interação entre dois fatores que favorecem a competência social: as características individuais e as estratégias utilizadas para a adaptação ao ambiente. A interação entre esses fatores e os recursos disponíveis no ambiente, como o apoio familiar e social, 
contribuem para que a pessoa possa obter um resultado satisfatório na luta contra as adversidades. Dentro dessa perspectiva, Coble et al. (1996) destacam outro aspecto importante para a competência social: a capacidade da pessoa para confiar em seus semelhantes e utilizar suas habilidades para se engajar em relacionamentos interpessoais. De acordo com esses autores, os relacionamentos próximos podem fornecer apoio em situações estressantes.

Vários autores distinguem a amizade como um dos aspectos que contribuem para a competência social da criança (Ladd, Kochenderfer, \& Coleman, 1996; Newcomb \& Bagwell, 1996; Price \& Ladd, 1986). Relações de amizade podem proporcionar a aquisição de habilidades essenciais para o desenvolvimento sócio-cognitivo-emocional das crianças, servindo como um contexto socializador (Newcomb \& Bagwell, 1996). Além disso, os processos de amizade promovem recursos emocionais e instrumentais que aumentam a capacidade das crianças para enfrentar positivamente as demandas do ambiente, adaptando-se a ele (Ladd et al., 1996).

Do ponto de vista da abordagem ecológica, as relações de amizade e a adaptação a diversos ambientes são permeadas pelas transições ecológicas (Bronfenbrenner, 1979/1996). As transiçõos ecológicas ocorrem quando a criança começa a ampliar suas relações, passando a frequientar outros microssistemas ecológicos, além do original (família). A transição da criança para outros microssistemas (escola, vizinhança) requer o exercício de novos papéis, específicos para cada contexto (Alves, 1997). A habilidade da criança para exercer novos papéis e adequar seu comportamento e atitudes a outros contextos é considerada um importante aspecto da competência social, uma vez que está relacionada com a adaptação a diferentes ambientes.

A empatia no relacionamento com pares e adultos é um aspecto que contribui para a competência social e transição ecológica da criança (Garmezy, 1996; Howes, Matheson, \& Hamilton, 1994). Uma criança socialmente competente é capaz de ser sensível e empática com seus pares, de se engajar em atividades sociais positivas, formar relações de amizade e adaptar-se em situações de stress (Howes et al., 1994). 
A empatia é definida como uma resposta emocional que deriva da percepção do estado ou condição de outra pessoa, sendo congruente com essa situação (Eisenberg \& Strayer, 1987). Empatia consiste em compartilhar uma emoção percebida de outra pessoa, sentindo a mesma emoção que ela está sentindo. É um sentimento diferente de simpatia e de angústia pessoal (personal distress), apesar de estar intimamente relacionado a estes conceitos. Simpatia refere-se a uma intensa preocupação com o sofrimento de outra pessoa. Consiste em sentir por outra pessoa e, geralmente, refere-se a sentimentos de tristeza ou lamento. Angústia pessoal, por outro lado, refere-se à experiência de um estado aversivo, como ansiedade ou preocupação, que não é congruente com o estado de outra pessoa e que leva a uma relação auto-orientada e egoísta (Eisenberg \& Strayer, 1987). Assim, a empatia é diferente da angústia pessoal, pois não é, em sua essência, uma resposta egoísta nem orientada para os outros, e da simpatia, que envolve uma clara orientação para terceiros.

O desenvolvimento da empatia é considerado por alguns autores como relacionado à expressividade emocional (Roberts \& Strayer, 1996). Empatia está associada com a habilidade para experienciar e expressar tanto emoções positivas como negativas. Crianças que experienciam emoções como medo, tristeza e felicidade, que reconhecem e aceitam estes sentimentos, e que conseguem manejar sua própria raiva durante as interações sociais, tendem a ser mais empáticas (Roberts \& Strayer, 1996).

A socialização das emoções é um aspecto de central importância para a expressividade emocional e empatia. A partir das experiências afetivas das crianças com seus pais e irmãos, estas aprendem a lidar com seus sentimentos e expressá-los (Eisenberg, Fabes, Schaller, Carlo, \& Miller, 1991). Garner, Jones e Miner (1994) referem que o apoio dos pais diante das emoções negativas dos filhos facilita a regulação da emoção e o reconhecimento das emoções de outros em diferentes contextos. Para alguns autores, crianças tendem a se comportar de uma maneira construtiva e demonstrar competência social quando elas aprendem a regular suas emoções (Eisenberg, Fabes, \& Murphy, 1996). Um estudo sobre a expressividade emocional (Luthar, 1991) 
demonstrou que a habilidade para regular emoções e expressá-las pode funcionar como um fator de proteção para a criança, resultando na promoção de competência social através da formação de vínculos de amizade.

Vários indícios apontam para uma possível relação entre a empatia e a competência social das crianças e sua influência na resiliência (Garmezy, 1996; Howes et al., 1994). O objetivo deste estudo foi avaliar a competência social e a empatia em crianças em situação de risco, visto que há uma carência na literatura da área de estudos com essa parcela da população. Entende-se por crianças em situação de risco pessoal e social aquelas cujo desenvolvimento não ocorre de acordo com o esperado para a sua faixa etária, dentro dos parâmetros de sua cultura (Bandeira, Koller, Hutz, \& Forster, 1996). Nesse estudo, o principal fator de risco foi a condição de pobreza das crianças.

\section{Método}

\section{Participantes}

Participaram deste estudo 100 crianças (50 meninos e 50 meninas), com idade entre seis e nove anos $(\mathrm{m}=7,81 ; \mathrm{dp}=0,86)$, estudantes de primeira a terceira séries de escolas públicas de Porto Alegre. O número de crianças em cada escola e a distribuição por sexo foi semelhante: na escola A, 22 crianças eram do sexo masculino e 27 eram do sexo feminino; na escola B, 23 crianças eram do sexo feminino e 28 eram do sexo masculino.

Os critérios para a seleção dos participantes foi a exposição à condição de pobreza (renda familiar abaixo de três salários mínimos) e o local de residência das crianças (favelas próximas às escolas onde o tráfico de drogas e o índice de violência - assaltos, assassinatos eram altos).

\section{Instrumentos e Procedimentos}

Determinada a população a ser estudada, as escolas foram contatadas após uma visita inicial à Secretaria de Educação do Município feita pela equipe do Centro de Estudos Psicológicos sobre Meninos e Meninas de Rua (CEP-RUA/UFRGS), objetivando o consenti- 
mento informado para a realização da pesquisa. A partir desse primeiro contato, foram realizadas reuniões com a equipe de direção e professores da escola para explicitar os objetivos do estudo, os procedimentos a serem tomados pela equipe de pesquisadores, bem como estabelecer que crianças participariam da pesquisa. $\mathrm{O}$ contato com os pais e/ou responsáveis pelas crianças foi realizado durante as reuniões de entrega de boletins, ocasião em que foi solicitado consentimento verbal para que seus filhos participassem da pesquisa, além de esclarecer os objetivos da mesma.

Os instrumentos utilizados com as crianças, nesse estudo, foram uma entrevista inicial, o Teste das Histórias Incompletas (Mondell \& Tyler, 1981) e a Escala de Empatia (Bryant, 1982), descritos a seguir.

\section{Entrevista inicial}

O primeiro contato feito com as crianças foi através de uma entrevista inicial, que tinha como objetivo estabelecer vínculo e, ao mesmo tempo, coletar dados sócio-demográficos da criança e de sua família.

\section{Teste das Histórias Incompletas}

A avaliação da competência social foi realizada através do Teste das Histórias Incompletas, de Mondell e Tyler (1981), adaptado para o uso com esta população no Brasil por Marques, Martins, Krum, Raymundo, Hoppe e Koller (1997). O instrumento foi traduzido do inglês para o português e alguns termos originais foram substituídos por outros, mais adequados ao contexto cultural brasileiro e à realidade dos participantes da pesquisa. Foram incluídas, também, ilustrações para cada uma das histórias, com o objetivo de estimular a participação e facilitar a verbalização das crianças.

$\mathrm{O}$ teste consiste em quinze histórias incompletas que envolvem interações entre crianças e seus pais, professores, colegas e irmãos, que são lidas para as crianças e a elas é solicitado que atribuam um final. Estas histórias são divididas em três subescalas que avaliam características importantes para a competência social: (1) confiança, (2) auto-eficácia e (3) iniciativa (Mondell \& Tyler, 1981). Uma das histórias que avaliam a confiança foi: "Marcos ficou furioso porque seu 
novo carro de brinquedo quebrou enquanto ele brincava. "Por que meus brinquedos preferidos sempre quebram?", ele pensou. Neste momento, Sérgio, seu irmão mais velho, entrou e perguntou qual era o problema. Então, Marcos explicou como o seu carro tinha quebrado. Sérgio disse: "Não se aborreça. Eu posso consertar seu carrinho amanhã". "Eu espero que tu consigas", pensou Marcos. No dia seguinte, depois da escola, Marcos viu Sérgio com uma faca e o carrinho em sua mão. O que você acha que aconteceu?". Uma das histórias que avaliam a auto-eficácia foi: "Todo mundo chamava ela de "Fernanda Sortuda" porque as coisas boas pareciam sempre acontecer com ela. Todos sabiam que Fernanda era a favorita na aula de Educação Física da escola, onde seu pai era professor. Mas, Fernanda também praticava muita ginástica, mais do que qualquer outra pessoa. Um dia, seu pai organizou uma competição de ginástica na aula. Era uma competição só para alunos da turma. Adivinha quem venceu? Certo, foi Fernanda. Porque você acha que isso aconteceu?" Uma das histórias que avaliam a iniciativa foi: "O irmão mais novo de Mariana, Frederico, vai fazer aniversário daqui a uma semana e Mariana ainda não tem um presente para ele. Ela queria dar um presente surpresa para ele, mas ela não sabe o que ele gostaria de ganhar ou como fazer para conseguir um presente sem que ele fique sabendo. Mariana pensou: "Como posso conseguir um presente para o Frederico sem que ele veja?" O que você acha que aconteceu a seguir?".

Com relação à avaliação, as histórias podem ser pontuadas com dois, um, ou zero pontos, de acordo com o Manual de Pontuação (Mondell \& Tyler, 1981), também adaptado para o uso com esta população por Marques, Martins, Krum, Raymundo e Koller (1997). Na subescala de confiança, os finais considerados mais competentes são aqueles com maior grau de otimismo e confiança interpessoal. Finais pessimistas e destrutivos são considerados menos competentes. $\mathrm{Na}$ subescala de auto-eficácia, os finais mais competentes são aqueles em que os esforços e comportamentos da criança são vistos como responsáveis pelos resultados. Finais menos competentes são aqueles em que a sorte ou forças externas às crianças são vistas como responsáveis pelos resultados. Na subescala de iniciativa, os finais 
nos quais a criança toma, ativamente, alguma decisão e se esforça para resolver o problema proposto são vistos como mais competentes. Finais menos competentes são aqueles em que a criança decide não tomar alguma atitude, desiste, ou permite que outra pessoa resolva o problema. As respostas mais competentes são avaliadas com dois pontos, respostas mistas ou ambíguas recebem um ponto e respostas não competentes recebem zero. A soma dos escores de cada subescala pode totalizar dez pontos, e a soma do escore de competência total pode totalizar trinta pontos.

As respostas atribuídas pelas crianças no teste foram analisadas individualmente por dois avaliadores treinados para esta tarefa. Posteriormente, foram feitas reuniões com o objetivo de comparar os resultados com relação à pontuação e obter consenso. Quando não houve concordância entre os dois avaliadores, um terceiro avaliador igualmente capacitado intervinha no processo e participava da discussão até obter consenso.

A análise de fidedignidade do Teste das Histórias Incompletas revelou um coeficiente alpha de Cronbach de 0,50, valor considerado baixo para a fidedignidade. Acredita-se, contudo, que esse resultado deve-se ao número reduzido de itens em cada subescala (cinco).

\section{Escala de Empatia}

A empatia foi avaliada através da Escala de Empatia (Bryant, 1982), adaptada para a utilização no Brasil com crianças de nível sócio-econômico baixo (Ribeiro, Koller, \& Camino, no prelo). Consiste em 22 itens afirmativos e negativos, os quais são lidos para a criança, que deve concordar ou não. Respostas empáticas são pontuadas com um ponto, respostas não empáticas são pontuadas com zero pontos. Assim, quanto maior o número de pontos obtidos, maior o nível de empatia. Com relação à fidedignidade da escala, foi encontrado um alpha de Cronbach de 0,54.

\section{Resultados}

Os dados obtidos com o Teste das Histórias Incompletas, para a competência social, foram analisados, separadamente, para cada 
subescala e para a escala de competência total. As médias e os desvios-padrão para cada uma delas são apresentados na Tabela 1.

Tabela 1.

Médias e Desvios-Padrão de Competência Social Total e Subescalas $(N=100)$

\begin{tabular}{lcccc}
\hline Escalas & $\begin{array}{c}\text { Escore } \\
\text { mínimo }\end{array}$ & $\begin{array}{c}\text { Escore } \\
\text { máximo }\end{array}$ & Média & $\mathrm{dp}$ \\
\hline Confiança & 0 & 10 & 4,59 & 2,17 \\
Auto-Eficácia & 2 & 10 & 6,72 & 1,88 \\
Iniciativa & 1 & 10 & 5,98 & 1,83 \\
Competência & 9 & 27 & 17,29 & 4,06 \\
Total & & & & \\
\hline
\end{tabular}

Uma análise de variância (ANOVA) demonstrou diferença entre os sexos, sendo que as meninas obtiveram melhores resultados do que os meninos (ver Tabela 2). Houve diferença significativa nas escalas de confiança $(\mathrm{F}(1,98)=5,76 ; \mathrm{p}<0,05)$, auto-eficácia $(\mathrm{F}(1,98)=4,24$; $\mathrm{p}<0,05)$, e competência total $(\mathrm{F}(1,98)=8,65 ; \mathrm{p}<0,05)$, demonstrando que as meninas tendem a ser mais competentes socialmente do que os meninos. Na escala de iniciativa essa diferença não foi encontrada.

Tabela 2.

Diferenças de sexo para a Competência Social: Médias e DesviosPadrão $(N=100)$

\begin{tabular}{lcccc}
\hline Escalas & Sexo & Médias & $\mathrm{dp}$ & $\mathrm{F}(1,98)$ \\
\hline Confiança & $\mathrm{M}$ & 4,08 & 2,13 & $5,76^{*}$ \\
& $\mathrm{~F}$ & 5,10 & 2,12 & \\
Auto-Eficácia & $\mathrm{M}$ & 6,34 & 2,01 & $4,24^{*}$ \\
& $\mathrm{~F}$ & 7,10 & 1,66 & \\
Iniciativa & $\mathrm{M}$ & 5,72 & 1,86 & 2,05 \\
& $\mathrm{~F}$ & 6,24 & 1,77 & \\
Competência & $\mathrm{M}$ & 16,14 & 4,00 & $8,65^{*}$ \\
Total & $\mathrm{F}$ & 18,44 & 3,81 & \\
${ }^{*} \mathrm{p}<0,05$ & & & &
\end{tabular}


Uma ANOVA revelou diferença significativa com relação às idades $(F(1,98)=13,44 ; p<0,05)$ na subescala de iniciativa. Crianças mais velhas ( $8 / 9$ anos; $n=64)$ possuem maior iniciativa do que crianças mais jovens ( $6 / 7$ anos; $n=36)$. A Tabela 3 apresenta as médias e os desviospadrão de competência social para os dois grupos etários.

Tabela 3.

Diferença de idade para a Competência Social: Médias e DesviosPadrão $(N=100)$

\begin{tabular}{lcccc}
\hline Escalas & $\begin{array}{c}\text { Idades (em } \\
\text { anos) }\end{array}$ & Médias & $\mathrm{dp}$ & $\mathrm{F}(1,98)$ \\
\hline Confiança & $6 / 7$ & 4,13 & 2,04 & 2,45 \\
& $8 / 9$ & 4,84 & 2,21 & \\
Auto-Eficácia & $6 / 7$ & 6,27 & 1,87 & 3,20 \\
& $8 / 9$ & 6,96 & 1,84 & \\
Iniciativa & $6 / 7$ & 5,13 & 1,80 & $13,44^{*}$ \\
& $8 / 9$ & 6,45 & 1,67 & \\
Competência & $6 / 7$ & 15,55 & 4,02 & $11,31^{*}$ \\
Total & $8 / 9$ & 18,26 & 3,76 & \\
\hline *p<0,05 & & & &
\end{tabular}

Conforme observado na Tabela 3 , há uma tendência para o aumento da competência social na medida em que aumenta a idade em todas as escalas, entretanto, somente nas escalas de iniciativa $(F(1,98)=13,44 ; \mathrm{p}<0,05)$ e competência total $(\mathrm{F}(1,98)=11,31 ; \mathrm{p}<0,05)$ essa diferença revelou-se significativa. Esse fato demonstra que crianças mais velhas tendem a ser mais competentes socialmente do que crianças mais jovens.

Com relação à empatia, a média encontrada para essa amostra foi de 13,24 (dp=3,12). A diferença entre os sexos na empatia foi significativa $(\mathrm{F}(1,98)=10,86 ; \mathrm{p}<0,05)$. Uma ANOVA demonstrou que as meninas $(\mathrm{m}=14,22 ; \mathrm{dp}=2,89)$ tendem a ser mais empáticas do que os meni$\operatorname{nos}(\mathrm{m}=12,26 ; \mathrm{dp}=3,05)$.

Diferenças de empatia entre grupos etários, entretanto, não foram encontradas nessa amostra. Apesar dos dados apontarem uma média 
numericamente mais alta para as crianças mais velhas $(8 / 9$ anos; $\mathrm{n}=36$; $\mathrm{m}=13,42 ; \mathrm{dp}=3,04)$ do que para as mais novas $(6 / 7$ anos; $\mathrm{n}=64 ; \mathrm{m}=12,91$; $\mathrm{dp}=3,25)$, essa diferença não é estatisticamente significativa.

Para avaliar a relação entre as três subescalas e a escala total da competência social e a escala de empatia foi calculado o Coeficiente de Correlação de Pearson (ver Tabela 4).

Tabela 4.

Correlação entre Competência Social e Empatia $(N=100)$

\begin{tabular}{lcccc}
\hline & Confiança & $\begin{array}{c}\text { Auto- } \\
\text { eficácia }\end{array}$ & Iniciativa & $\begin{array}{c}\text { Competência } \\
\text { Total }\end{array}$ \\
\hline Empatia & 0,13 & $0,26^{*}$ & 0,02 & $0,21^{*}$ \\
\hline${ }^{*}<0,05$ & & & &
\end{tabular}

A análise demonstra que há correlação significativa da escala de auto-eficácia e empatia $(r=0,26 ; p<0,05)$ e da escala de competência total e empatia $(r=0,21 ; p<0,05)$. Esse dado revela que, quanto mais empática é uma criança, mais competente socialmente ela tende a ser.

\section{Discussão}

Apesar da evidência de risco presente na condição de pobreza e no local de moradia ser um fator constante na amostra, os achados quando comparados a resultados obtidos na literatura (Bryant, 1982; Mondell \& Tyler; 1981), com crianças de nível sócio-econômico médio, demonstram que o grupo avaliado não difere em suas estratégias para adaptação em situações estressantes. Com relação à competência social, o grupo estudado por Mondell e Tyler (1981) apresentou média de 17,75 (dp=4,20), resultado semelhante ao encontrado nessa pesquisa (ver Tabela 1). Ao comparar as médias totais obtidas nessa amostra com as do estudo de Mondell e Tyler através do teste t de Student para uma média populacional, observou-se que não há diferença significativa entre as duas amostras. A análise isolada de cada subescala, entretanto, revela resultados diferenciados. Com relação à subescala de confiança, a média obtida nesse estudo e no estudo de 
Mondell e Tyler não são estatisticamente diferentes. As médias das subescalas de auto-eficácia $(\mathrm{m}=6,17 ; \mathrm{dp}=2,17)$ e de iniciativa $(\mathrm{m}=7,04$; $\mathrm{dp}=2,08)$, contudo, são diferentes estatisticamente. A média de autoeficácia desse grupo é superior a do estudo de Mondell e Tyler, e a média de iniciativa é inferior a deles (ver Tabela 1). Em seu estudo, Mondell e Tyler observaram que a baixa pontuação na escala de iniciativa estava presente nos grupos menos competentes, em contrapartida, alta pontuação nas escalas de confiança e auto-eficácia estavam presentes nos grupos mais competentes. Esse fato era o que basicamente distinguia os grupos mais competentes dos grupos menos competentes. Os grupos mais competentes demonstravam mais sentimentos de controle pessoal e responsabilidade sobre os eventos de suas vidas, ao passo que os grupos menos competentes demonstravam mais passividade ao enfrentar as situações adversas. A diferença significativa entre as médias da escala de iniciativa nos dois estudos parece ser um indício de que a variável risco, presente nessa pesquisa, possa estar influenciando na capacidade de interagir com os eventos de vida e interferindo no desenvolvimento de estratégias para ação. Coble et al. (1996) enfatizam a importância da capacidade de iniciativa para a competência social. Os autores atribuem aos comportamentos de busca de solução de problemas um papel fundamental na determinação do sucesso na adaptação em situações adversas.

A capacidade para a iniciativa, entretanto, foi observada com um melhor desempenho de crianças mais velhas dessa amostra. Os resultados apontam para uma diferença significativa entre as idades, sendo que as crianças mais velhas (8/9 anos) obtiveram um melhor desempenho do que as crianças mais novas (6/7 anos) nas escalas de iniciativa e competência total (ver Tabela 3). Esse achado sugere que existe diferença entre idades para a competência social, corroborando a literatura (Kliewer, 1991; LaFreniere \& Dumas, 1996; Mondell \& Tyler, 1981). Kliewer (1991) refere que essa diferença é esperada porque crianças mais jovens possuem menos estratégias em seu repertório para lidar com situações adversas do que adolescentes e adultos, pois ainda estão desenvolvendo suas habilidades cognitivas e sua experiência é limitada, comparada à de uma criança mais madura. 
A análise da diferença entre os sexos para a competência social nessa amostra demonstra que as meninas obtiveram melhores resultados do que os meninos nas escalas de confiança, auto-eficácia e competência total, sendo mais competentes socialmente (ver Tabela 2). Esse achado revela uma diferença entre os sexos para a competência social, embora não corrobore aos resultados encontrados por Mondell e Tyler (1981). Esses autores não encontraram diferença significativa entre os sexos, contudo, observaram que as meninas pontuaram consistentemente mais alto do que os meninos. Um estudo recente sobre competência social e avaliação de comportamento traz dados complementares aos desse estudo, demonstrando que as meninas são mais competentes socialmente e apresentam menos problemas de comportamento durante a adaptação à escola do que os meninos (LaFreniere \& Dumas, 1996). Essa diferença sugere que a variável sexo esteja associada com a capacidade para adaptação em situações de stress. De acordo com LaFreniere e Dumas (1996), é provável que algumas crianças respondam a situações estressantes manifestando comportamentos disruptivos, e existem evidências na literatura de que esse tipo de comportamento é mais freqüente em meninos (Zahn-Waxler, Cole, \& Barrett, 1991).

Dentro da Psicopatologia do Desenvolvimento há uma distinção comum entre os autores com relação à direção da manifestação do sintoma: os sintomas direcionados ao comportamento - externados (agressividade e uso de drogas, por exemplo) (Short \& Brokaw, 1994) e os sintomas autodirecionados - internalizados (depressão e ansiedade, por exemplo) (Bucy, 1994) que podem explicar esses achados. Zahn-Waxleret al. (1991) afirmam que o desenvolvimento dos sintomas direcionados ao comportamento são mais freqüentes em meninos e os sintomas auto-direcionados são mais comuns em meninas. Essa tendência na manifestação de sintomas revela que todas as pessoas que vivenciam situações de stress sofrem com essas adversidades, entretanto, algumas pessoas tendem a demonstrar seu sofrimento através do comportamento, outras aparentemente não demonstram sofrimento, parecendo estarem adaptadas à situação. Esse é o caso que mais freqüentemente acontece com as meninas, que, por serem em 
geral mais empáticas e menos agressivas do que os meninos, parecem mais adaptadas e mais competentes nos seus relacionamentos sociais (Zahn-Waxler et al., 1991).

A avaliação da empatia nessa amostra revela uma diferença significativa entre os sexos, sendo as meninas mais empáticas do que os meninos, um achado que, de um modo geral, corrobora a literatura. $\mathrm{O}$ estudo de Bryant (1982) com crianças e adolescentes demonstrou diferença entre os sexos, sendo as meninas mais empáticas. A revisão de literatura realizada por Lennon e Eisenberg (1987) com relação às diferenças de sexo e de idade para a empatia, entretanto, traz dados controversos. Esses pesquisadores analisaram diferentes estudos e concluíram que a falta de consenso com relação às diferenças de sexo para a empatia é um fator decorrente do tipo de metodologia utilizada para avaliar a mesma. Alguns estudos utilizam técnicas como gravuras e histórias, outros, medidas de avaliação facial ou vocal, outros ainda, utilizam questionários. De acordo com os autores, os estudos que utilizam questionários são os mais consistentes em relação à diferença entre os sexos, demonstrando que as meninas são mais empáticas do que os meninos (Lennon \& Eisenberg, 1987). O fato das meninas demonstrarem maiores níveis de empatia do que os meninos em procedimentos como questionários é explicado por alguns autores devido às práticas de socialização na família (Zahn-Waxler et al., 1991). Atitudes como cuidar e confortar estão mais ligadas ao papel da mãe, assim, passa a ser internalizado com maior frequiência pelas meninas, através da identificação, que desenvolvem características como preocupação e interesse pelos outros mais do que os meninos (ZahnWaxler et al., 1991).

Diferenças de empatia entre idades não são observadas nessa amostra, em oposição aos achados de Bryant (1982). Em seu estudo realizado com crianças e adolescentes de primeira, quarta e sétima séries, foi encontrada diferença significativa entre as idades, sendo que os adolescentes de sétima série demonstraram ser mais empáticos do que as crianças de primeira e quarta séries. Do mesmo modo, a revisão feita por Lennon e Eisenberg (1987), com relação à diferença de idade para a empatia em procedimentos como questionário apre- 
sentou dados similares. Em suma, esses autores concluíram que a empatia aumenta durante os primeiros anos escolares pelo menos com relação às pessoas do mesmo sexo, entretanto, nenhuma relação aparente entre idade e empatia foi observada em crianças com mais de onze anos. Apesar desses achados, os autores referem que os dados das pesquisas sobre empatia são difíceis de interpretar devido à variabilidade das emoções envolvidas durante a mensuração (Lennon \& Eisenberg, 1987).

O nível de risco vivenciado pelas pessoas dessa amostra tende a ser, entretanto, amenizado pela correlação entre dois fatores de proteção: a competência social e a empatia. A relação entre essas duas variáveis demonstra que, quanto mais empática é uma criança, mais competente socialmente ela é capaz de ser. Esse achado corrobora estudos anteriores, que revelam que crianças socialmente competentes tendem a ser sensíveis e empáticas com seus pares, capazes de estabelecer relações de amizade e habilidosas na resolução de problemas (Howes et al., 1994). Uma vez que a empatia favorece o vínculo entre as pessoas, espera-se que ela contribua para o desenvolvimento das relações de amizade. As relações de amizade, em situações de stress, funcionam como apoio social, e auxiliam na capacidade de adaptação (Ladd et al., 1996).

De um modo geral, os resultados obtidos sobre a competência social e a empatia de crianças em situação de pobreza destacam a importância desses dois fatores para o desenvolvimento de estratégias para a adaptação a situações adversas, favorecendo a resiliência e reduzindo a vulnerabilidade. Trabalhar no sentido de incrementar os recursos disponíveis, tanto em nível individual, quanto em nível ambiental, pode vir a ser uma maneira de minimizar os danos causados pelas adversidades. Proporcionar situações nos mais variados contextos (familiar, escolar etc.) para o desenvolvimento de características como empatia e competência social, assim como oferecer condições para o estabelecimento de uma rede de apoio social são formas de favorecer a resiliência. Da mesma forma, o estabelecimento de uma rede social de apoio à família consiste numa maneira de trabalhar contra a miséria afetiva, cujos prejuízos são mais avassaladores dos que os da própria miséria econômica. Assim, a relevância de estudos nes- 
sa área reside na possibilidade de implementação de programas que auxiliem no desenvolvimento de características que contribuam para a resiliência, como competência social e empatia.

\section{Agradecimentos}

As autoras agradecem a participação dos bolsistas de Iniciação Científica do Grupo de Estudos sobre Competência Social e Resiliência do CEP-RUA/UFRGS. Apoio CNPq.

\section{Referências}

Alves, P. B. (1997). A ecologia do desenvolvimento humano: experimentos naturais e planejados - Resenha. Psicologia: Reflexão e Crítica, 10, 369-373.

Bandeira, D., Koller, S. H., Hutz, C., \& Forster, L. (1996). Desenvolvimento psico-social e profissionalização: uma experiência com adolescentes de risco. Psicologia: Reflexão e Crítica, 9, 185-207.

Bronfenbrenner, U. (1996). A ecologia do desenvolvimento humano: experimentos naturais e planejados. Porto Alegre: Artes Médicas. (Original publicado em 1979)

Bronfenbrenner, U. \& Morris, P. (1998). The ecology of developmental processes. In W. Damon (Org.), Handbook of child psychology (v.1, pp.9931027). New York: John Wiley \& Sons.

Bryant, B. K. (1982). An index of empathy for children and adolescents. Child Development, 53, 413-425.

Bucy, J. E. (1994). Internalizing affective disorders. In R.J. Simmeonson (Org.), Risk, resilience, and prevention: promoting the well-being of all children (pp.219-238). Baltimore: Paul H. Brooks.

Cecconello, A. M. (1999). Competência social, empatia e representação mental da relação de apego em famílias em situação de risco. Dissertação de Mestrado Não-Publicada. Curso de Pós-Graduação em Psicologia do Desenvolvimento. Universidade Federal do Rio Grande do Sul. Porto Alegre, RS.

Coble, H. M., Gantt, D. L., \& Mallinckrodt, B. (1996). Attachment, social competency, and the capacity to use social support. In G. R. Pierce, B. R. Sarason, \& I. G. Sarason (Orgs.), Handbook of social support and the family (pp.141-172). New York: Plenum Press.

Del Prette, Z. A. P., \& Del Prette, A. (1999). Psicologia das habilidades sociais: terapia e educação. Rio de Janeiro: Vozes.

Eisenberg, N., Fabes, R. A., \& Murphy, B. C. (1996). Parents' reactions to children's negative emotions: relations to children's social competence and comforting behavior. Child Development, 67, 2227-2247. 
Eisenberg, N., Fabes, R. A., Schaller, M., Carlo, G., \& Miller, P. A. (1991). The relations of parental characteristics and practices to children's vicarious emotional responding. Child Development, 62, 1393-1408.

Eisenberg, N. \& Strayer, J. (1987). Critical issues in the study of empathy. In N. Eisenberg \& J. Strayer (Orgs.), Empathy and its development (pp. 313). Cambridge: Cambridge University Press.

Emde, R. N. (1994). Individuality, context, and the search for meaning (Presidential Address). Child Development, 65, 719-737.

Fischer, C. B. (1993). Integrating science and ethics in research with high-risk children and youth. Social Policy Report, 7(4), 1-24.

Garmezy, N. (1996). Reflections and commentary on risk, resilience, and development. In R. J. Haggerty, L. R. Scherrod, N. Garmezy \& M. Rutter, (Orgs.), Stress, risk, and resilience in children and adolescentes: processes, mechanisms, and interventions (pp.1-18). Cambridge: Cambridge University Press.

Garmezy, N. \& Masten, A. (1994). Chronic Adversities. In M. Rutter, E. Taylor, \& L. Herson (Orgs.), Child and Adolescent Psychiatry (pp. 191207). Oxford: Blackwell Scientific.

Garner, P. W., Jones, D. C., \& Miner, J. L. (1994). Social competence among low-income preschoolers: emotion socialization practices and social cognitive correlates. Child Development, 65, 622-637.

Howes, C., Matheson, C. C., \& Hamilton, C. E. (1994). Maternal, teacher, and child care history correlates of children's relationships with peers. Child Development, 65, 264-273.

Huston, A. C., McLoyd, V. C., \& Coll, C. G. (1994). Children in poverty: issues in contemporary research. Child Development, 65, 275-282.

Hutz, C. S., \& Koller, S. H. (1997). Questões sobre o desenvolvimento de crianças em situação de rua. Estudos de Psicologia, 2, 175-197.

Hutz, C. S., Koller, S. H., \& Bandeira, D. R. (1996). Resiliência e vulnerabilidade em crianças em situação de risco. Coletâneas da ANPEPP, 1(12), 79-86.

Kliewer, W. (1991). Coping in middle childhood: relations to competence, type A behavior, monitoring, blunting, and locus of control. Developmental Psychology, 27, 689-697.

Ladd, G. W., Kochenderfer, B. J., \& Coleman, C. C. (1996). Friendship quality as a predictor of young children's early school adjustment. Child Development, 67, 1103-1118.

LaFreniere, P. J., \& Dumas, J. E. (1996). Social competence and behavior evaluation in children ages 3 to 6 years: the short form (SCBE-30). Psychological Assessment, 8, 369-377.

Lennon, R., \& Eisenberg, N. (1987). Gender and age differences in empathy and sympathy. In N. Eisenberg \& J. Strayer (Orgs.), Empathy and its development (pp.195-217). Cambridge: Cambridge University Press. 
Luthar, S. S. (1991). Vulnerability and resilience: a study of high risk adolescents. Child Development, 62, 600-616.

Marques, A. L., Martins, C. B., Krum, F. M. B., Raymundo, M. B., \& Koller, S. H. (1997). Adaptação do manual de pontuação do Teste das Histórias Incompletas - THI. Manuscrito Não-Publicado. Curso de Pós-Graduação em Psicologia do Desenvolvimento. Universidade Federal do Rio Grande do Sul. Porto Alegre, RS.

Marques, A. L., Martins, C. B., Krum, F. M. B., Raymundo, M. B., Hoppe, M. W., \& Koller, S. H. (1997, Maio). Adaptação do Teste das Histórias Incompletas - THI. Trabalho apresentado no VII Encontro Nacional de Testes Psicológicos e I Congresso Ibero-Americano de Avaliação Psicológica. Porto Alegre, RS.

Masten, A. S. \& Coatsworth, J. D. (1995). Competence, resilience and psychopathology. In D. Cicchetti \& D. Cohen (Orgs.), Developmental Psychopathology (v.2, pp.715-752). New York: Wiley Interscience.

Mondell, S., \& Tyler, F. B. (1981). Child psychosocial competence and its measurement. Journal of Pediatric Psychology, 6, 145-154.

Newcomb, A. F., \& Bagwell, C. L. (1996). The developmental significance of children's friendship relations. In W. M. Bukowski, A.F. Newcomb, \& W. W. Hartup (Orgs.), The company they keep: friendship in childhood and adolescence (pp.289-321). New York: Cambridge University Press.

Nunes, T. (1994). O ambiente da criança. Cadernos de Pesquisa, 89, 5-23.

Pierre, R. G., \& Layzer, J. I. (1998). Improving the life chances of children in poverty: assumptions and what we have learned. Social Policy Report, 12(4), 1-28.

Price, J. M., \& Ladd, G. W. (1986). Assessment of children's friendships: implications for social competence and social adjustment. In R. J. Prinz (Org.), Advances in behavioral assessment of children and families, (v.2, pp.121-149). Greenwich, CT: JAI Press.

Ribeiro, J., Koller, S. H., \& Camino, C. (no prelo). Adaptação da escala de empatia. Estudos em Psicologia (Campinas).

Roberts, W. \& Strayer, J. (1996). Empathy, emotional expressiveness, and prosocial behavior. Child Development, 67, 449-470.

Rutter, M. (1996). Psychosocial resilience and protective mechanisms. In J. Rolf, A. S. Masten, D. Cicchetti, K. H. Nuechterlein, \& S. Weintraub (Orgs.), Risk and protective factors in the development of psychopathology (pp.181-214). New York: Cambridge University Press.

Short, R. F., \& Brokaw, R. (1994). Externalizing behavior disorders. In R. J. Simmeonson (Org.), Risk, resilience and prevention: promoting the wellbeing of all children (pp.203-217). Baltimore: Paul H. Brooks Publishing.

Tyler, F. (1984). El comportamiento psicosocial, la competencia psicosocial individual y las redes de intercambio de recursos como ejemplos de psicologia comunitaria. Revista Latino-Americana de Psicologia, 16, 77-92. 
Zahn-Waxler, C., Cole, P. M., \& Barrett, K. C. (1991). Guilt and empathy: sex differences and implications for the development of depression. In J. Garber \& K. A. Dodge (Orgs.), The development of emotion regulation and dysregulation (pp.243-272). Cambridge: Cambridge University Press.

Zigler, E., \& Trickett, P. K. (1978). IQ, social competence, and evaluation of early childhood intervention programs. American Psychologist, 33, 789-798.

Zimmerman, M. A. \& Arunkumar, R. (1994). Resiliency research: implications for schools and policy. Social Policy Report, 8(1), 1-18.

Alessandra Marques Cecconello, psicóloga, doutoranda em Psicologia do Desenvolvimento do Curso de Pós-Graduação em Psicologia do Desenvolvimento da Universidade Federal do Rio Grande do Sul, membro do Centro de Estudos Psicológicos sobre Meninos e Meninas de Rua (CEP-RUA/UFRGS), desde 1996, é coordenadora do Núcleo de Estudos e Capacitação em Desenvolvimento Humano e da Equipe de Pesquisa sobre Competência Social e Resiliência do CEPRUA/UFRGS.
Sobre as autoras 
Sílvia Helena Koller, psicóloga, doutora em educação (PUC/ $\mathrm{RS}$ ), pesquisadora do $\mathrm{CNPq}$, é professora do Curso de Pós-Graduação em Psicologia do Desenvolvimento da Universidade $\mathrm{Fe}$ deral do Rio Grande do Sul. Coordenadora do Centro de Estudos Psicológicos sobre Meninos e Meninas de Rua (CEP-RUA/ UFRGS).

Endereço para correspondência: CEP-RUA/UFRGS, Instituto de Psicologia, Rua Ramiro Barcelos, 2600/104, 90035.003, Porto Alegre, RS. Fone: $\left(0^{* * 51)}\right.$ 316-5150. Fax: (0**51) 3304797.

E-mail:

cep_rua@vortex.ufrgs.br. 